\title{
Estilo de vida y factores socioeconómicos en estudiantes de electivas de actividad física y deporte de la Pontificia Universidad Javeriana*
}

\author{
Marlucio de Souza Martins*** \\ Aura Catalina Zea Robles*** \\ Gloria Consuelo Rodríguez Caro**** \\ Arturo Molina Pinzón****
}

Recibido: 23 de agosto de 2016 - Aprobado: 30 de septiembre de 2016

\section{Resumen}

El estudio tiene como finalidad correlacionar los estilos de vida y factores socioeconómicos con la salud de los estudiantes de las electivas de actividad física y deporte de la Pontificia Universidad Javeriana, sede Bogotá-Colombia. Es un estudio cuantitativo descriptivo, de corte transversal, con una muestra de 1410 estudiantes con edad entre 24 y 29 años que corresponde a 748 mujeres y 662 hombres. Fue aplicado un cuestionario estandarizado propuesto por Nahas (2013), que presenta los factores nutricionales, el estrés, la actividad física, el comportamiento preventivo y las relaciones sociales como componentes que afectan la salud de las personas y están asociados al desarrollo de las enfermedades crónicas no transmisibles. La menor calificación se encontró en los factores nutricionales, de actividad física y de conducta preventiva. Por otro lado, se encontró una mejor calificación en las relaciones sociales y el control del estrés. Los resultados socioeconómicos demuestran que los estudiantes tienen preferencia por deportes individuales; viven con los padres y familiares, ubicados en estrato de clase media, y se presenta un alto índice de padres con formación universitaria. Se concluye que existe una tendencia de estilos de vida no saludables que puede afectar negativamente la salud de esta población. Es importante desarrollar estrategias de intervención en el contexto social, para generar un cambio de conducta y adoptar un estilo de vida saludable para mejorar la calidad de vida.

Palabras clave: factores socioeconómicos, estilo de vida, actividad física, salud

Este artículo de investigación científica y desarrollo tecnológico expone los resultados de un proyecto realizado en el segundo semestre del 2015, sin financiamiento. DOI: http://dx.doi.org/10.15332/s0120-8454.2017.0090.10

* Magíster en Educación, Pontifica Universidad Javeriana, carrera 7. ${ }^{\text {a } \# ~ 40-62, ~ e d i f i c i o ~ 25, ~ p i s o ~ 4, ~ B o g o t a ́, ~ C o r r e o ~ e l e c-~}$ trónico: mdesouzamartins@javeriana.edu.co.

** Fisioterapeuta, Universidad Santo Tomás, carrera 9. \# \# 51-11, Bogotá. Correo electrónico: aurazea@usantotomas.edu.co.

Socióloga, Pontifica Universidad Javeriana. Correo electrónico: grodrig@javeriana.edu.co

Especialista en Ejercicio Físico para la Salud, Pontifica Universidad Javeriana. Correo electrónico: amolina@javeriana.edu.co 


\title{
Lifestyle and socioeconomic factors in students in the elective courses of physical activity and sport at Pontificia Universidad Javeriana*
}

\author{
Marlucio de Souza Martins** \\ Aura Catalina Zea Robles*** \\ Gloria Consuelo Rodríguez Caro**** \\ Arturo Molina Pinzón ****
}

\section{A bstract}

The objective of this study was to correlate the lifestyles and socioeconomic factors with the health of the students in the Pontificia Universidad Javeriana, Bogotá - Colombia. It's a quantitative descriptive cross-sectional study with a sample of 1410 students aged between 24 and 29 years corresponding to 748 women and 662 men. Was applied a standardized questionnaire proposed by Nahas (2013), It consist of nutritional factors, stress, physical activity, preventive behavior and social relations as components that affect the health of people, therefor they're associated with the development of noncommunicable chronic diseases. The lower ratings were the nutritional factors, physical activity and preventive behavior. On the other hand, was found a better rating in social relationships and stress management. In the socioeconomic results were evidenced that the students have a preference for individual sports; They live with their parents and relatives at middle-class houses and the educational level of their parents is university. We concluded that there is a trend of styles of unhealthy life that can adversely affect the health of this population. Is important to develop strategies of intervention in the social context, to generate a change in behavior and adopt a healthy lifestyle to improve the quality of life.

Keywords: Socioeconomic factors, lifestyle, physical activity, health.

This scientific research and technological development article presents the results of a project carried out in the second half of 2015, without funding. DOI: http://dx.doi.org/10.15332/s0120-8454.2017.0090.10

* Magister in Education, Pontifica Universidad Javeriana, mdesouzamartins@javeriana.edu.co - Carrera 7a \# 40-62, edificio 25 , piso 4 .

..- Physiotherapist, University of Santo Tomás, aurazea@usantotomas.edu.co - Carrera 9a \# 51-11.

.... Sociologist, Pontifica Universidad Javeriana, grodrig@javeriana.edu.co - Carrera 7a \# 40-62, edificio 25, piso 4.

..... Specialist in Physical Exercise for Health, Pontificia Universidad Javeriana, amolina@javeriana.edu.co - Carrera $7^{\mathrm{a}}$ \# 40-62, edificio 25 , piso 4 


\title{
Style de vie et facteurs socio-économiques chez les étudiants de matières optionnelles d'activité physique et sport de l’Université Javeriana*
}

\author{
Marlucio de Souza Martins** \\ Aura Catalina Zea Robles*** \\ Gloria Consuelo Rodríguez Caro**** \\ Arturo Molina Pinzón ${ }^{* * * *}$
}

\section{Résumé}

L'étude a pour but de mettre en rapport les styles de vie et les facteurs socioéconomiques avec la santé des étudiants des matières optionnelles d'activité physique et sport de l'Université Javeriana, à Bogotá en Colombie. C'est une étude quantitative descriptive, de sondage transversal, avec un échantillon de 1410 étudiants entre 24 et 29 ans qui correspondent à 748 femmes et 662 hommes. Un questionnaire standardisé proposé par Nahas (2013) a été appliqué, celui-ci présente les facteurs alimentaires, le stress, l'activité physique, le comportement préventif et les relations sociales comme des éléments qui affectent la santé des personnes et sont associés au développement de maladies chroniques non transmissibles. Le plus mauvais résultat se trouve dans les facteurs alimentaires, d'activité physique et de conduite préventive. D'un autre côté, on a obtenu de meilleurs résultats dans les relations sociales et le contrôle du stress. Les résultats socio-économiques démontrent que les étudiants préfèrent les sports individuels; qu'ils vivent avec leurs parents ou famille, qu'ils sont de classe moyenne, et un haut pourcentage de leurs parents ont une éducation universitaire. On conclut qu'il existe une tendance de modes de vie non sains qui peut affecter de manière négative la santé de cette population. Il est important de développer des stratégies d'intervention adaptées au contexte social, afin de modifier les comportements et adopter un mode de vie sain pour améliorer la qualité de vie.

Mots clés: Facteurs socioéconomiques, mode de vie, activité physique, santé.

Cet article de recherche scientifique et de développement technologique expose les résultats d'un projet réalisé pendant le deuxième semestre de 2015, sans financement.DOI: http://dx.doi.org/10.15332/s0120-8454.2017.0090.10

" Magister en Éducation, Université Javeriana, mdesouzamartins@javeriana.edu.co - Carrera 7. a \# 40-62, edificio 25, piso 4.

..*Physiothérapeute, Université Santo Tomás, aurazea@usantotomas.edu.co - Carrera 9. ${ }^{a}$ \# 51-11.

.... Sociologue, Université Javeriana, grodrig@javeriana.edu.co - Carrera 7. \# \# 40-62, edificio 25, piso 4.

..... Spécialiste en Exercice Physique pour la Santé, Université Javeriana, amolina@javeriana.edu.co - Carrera 7. \# 40-62, edificio 25 , piso 4 . 


\section{Introducción}

Actualmente se evidencia una creciente preocupación con el estilo de vida y las consecuencias que puedan traer en la salud de las personas. En el siglo XXI, existe una nueva generación que es reconocida como sociedad del milenio, debido a un proceso de modernización, globalización y desarrollo tecnológico que refleja nuevos estilos de vida en la sociedad. Esta tendencia denominada era tecnológica, en la cual las personas dedican cada vez menos tiempo a la actividad física y el deporte. Por lo anterior, se refleja el crecimiento del sedentarismo, y como consecuencia se genera la aparición de enfermedades crónicas no transmisibles, como la hipertensión arterial, la diabetes y la obesidad, entre otras, que tiene una relación directa con la inactividad física.

El American College of Sport Medicine (ACSM) define actividad física como cualquier movimiento corporal producido por la contracción músculo-esquelética que resulta en un aumento sustancial de los requerimientos del gasto energético en reposo (2014). Según Krupp y Chatton (2014), el consumo de tabaco es el primer factor de decesos en américa latina, seguido por la inactividad física, que aporta el $28 \%$ de las muertes relacionadas con enfermedades crónicas.

Otros estudios realizados por Vidarte et al. (2012), determinan que el nivel de sedentarismo tiene una prevalencia del $73 \%$ en la población general colombiana; por género, el nivel de sedentarismo en hombres atiende al $61 \%$ y en mujeres, al $84 \%$. Como resultado de este tipo de conducta sedentaria, en Colombia en el año 2008 se presentaron 109.405 muertes por enfermedades del aparato circulatorio, enfermedad isquémica del corazón, enfermedades cerebrovasculares y diabetes mellitus en personas mayores de 45 años (Garzón y Rodríguez, 2012).

Por lo anterior, el comportamiento sedentario y los bajos niveles de actividad física, se asocian con un mayor riesgo de enfermedades crónicas no transmisibles (ECNT). Conforme a la Organización Mundial de la Salud (OMS), "aunque el sedentarismo no afecta a la salud mental y psíquica, se sabe que la participación en actividades físicas puede reducir el grado de estrés, la ansiedad y los síntomas de depresión entre las personas que sufren estos trastornos" (2005, p. 5)

Por otro lado, el Departamento Administrativo Nacional de Estadística de Colombia (DANE), reporta que, entre 1990 y 2005 la mortalidad por ECNT aumentó del $59 \%$ al 62,6\%, mientras que las muertes por violencia y accidentes representaron el $23 \%$. Esto deja en evidencia, una problemática que cada vez empeora y que por lo cual, se deben tomar medidas para intentar disminuir la adquisición de estas ECNT. Asimismo, teniendo en cuenta las prioridades para la OMS, las cuales ayudan a identificar las acciones de intervención e impacto para la reducción de adquisición de ECNT, los factores de riesgo más asociados a estas enfermedades son, el tabaquismo, consumo de alcohol, sedentarismo y hábitos de alimentación no saludable (OMS, 2013). 
Asimismo, la salud humana está interconectada durante todo el ciclo vital; la concepción, vida fetal, primera infancia, adolescencia, la edad adulta y la tercera edad, cada una de estas etapas presenta sus propias necesidades y problemas de salud (Gluckman et al., 2008), hay que mencionar además que, investigaciones recientes han descrito cómo la infancia puede tener un profundo impacto sobre la salud y la enfermedad en la edad adulta, teniendo especial cuidado en períodos críticos, en los que se promueve el desarrollo de la obesidad, como son: el periodo prenatal, la primera infancia y la adolescencia (Guoying et al., 2013).

En contraste con lo anterior, se ha observado que la población en general todavía no es consciente de las implicaciones que traen la práctica de hábitos no saludables, como el sedentarismo, alimentación no adecuada y el consumo de tabaco, que se sabe aumenta el riesgo de desarrollar ECNT, al punto que, se ha convertido en una problemática para países subdesarrollados OMS (2008), donde cerca de 36 millones de muertes fueron causadas por ECNT, comprende principalmente, enfermedades cardiovasculares (48 \%), cáncer $(21 \%)$, enfermedades respiratorias crónicas (12\%) y diabetes (3,5\%); a su vez, en el 2008 se reportaron 57 millones de muertes correspondientes al $63 \%$ de la población mundial (Arestedt et al., 2014).

Con relación a lo anterior, se ha evidenciado que, a finales del Siglo XX, los factores de riesgo cardiovascular en población juvenil no presentaban investigaciones masivas y específicas, que relacionaran los efectos de la práctica de estilos de vida no saludables con las ECNT. Sin embargo, recientemente se ha encontrado que los adolescentes están presentando conductas que afectan su estado de salud, las cuales han sido adquiridas en etapas como la primera infancia tendiendo a persistir hasta la edad adulta (Zea et al., 2014).

Teniendo en cuenta lo anterior, Hernández, Oliva y Pertegal (2013) describe lo importante que es la primera infancia y la adolescencia para el establecimiento de los hábitos de vida, sobre todo en la adolescencia, en donde se presentan importantes cambios psicológicos y contextuales, en los que los adolescentes realizan sus primeras salidas y reuniones con sus iguales, sin control de un adulto y en los que van ganando autonomía para tomar decisiones relacionadas con el ocio y el estilo de vida. Moscoso (2013) afirma que el 89 \% de los jóvenes universitarios presenta hábitos de alimentación deficiente y el $46 \%$ realiza actividad física baja, lo que resulta, una predisposición al desarrollo de ECTN.

Por otra parte, con respecto a la tendencia evolutiva que siguen los estilos de vida, varias investigaciones realizadas en España muestran cómo a medida que avanza la etapa de adolescencia se va produciendo un continuo déficit del estilo de vida saludable (Batista, Fortiana y Currie, 2004).

Para el estudio de los estilos de vida de los adolescentes es determinante comprender el contexto familiar, social y factores socioeconómicos que les permea. Desde una visión sociológica, los estilos de vida son comprendidos como 
patrones de actividades o conductas que las personas eligen adoptar entre aquellas que están disponibles en función de su contexto social (Bandura, 1977).

Dicho de otra manera, los estilos de vida son la forma en que las relaciones sociales son reproducidas a través de actos y actores concretos. Allí las posiciones sociales se conciben esquemas o principios de percepción, de acción y de formas de sentir, en el que se construye las experiencias de los grupos sociales a partir de trayectorias comunes que le son propias y en donde se califican según la clase social como "distinguidos" "vulgares" etc. (Bourdieu, 1998).

Continuando con la idea, el hecho de estar inmersos en un contexto social condiciona en mayor o menor grado, para Bandura (1977), los adolescentes tienen características diferentes, de manera que es posible analizar la dimensión de las conductas que conforman los estilos de vida saludables en esta etapa evolutiva. Si los adolescentes ven consecuencias deseables y positivas en la conducta observada, es más probable que la imiten, la tomen como modelo y la adopten.

El objetivo fundamental de esta investigación fue correlacionar los estilos de vida y factores socioeconómicos con la salud de los estudiantes de las electivas de actividad física y deporte de la Pontificia Universidad Javeriana, sede Bogotá - Colombia. A partir de esta investigación, se recomienda estrategias para desarrollar estilos de vida saludables, a modo de compromiso individual y social, con miras a satisfacer las necesidades fundamentales, es decir, mejorar la calidad de vida y el desarrollo humano de los individuos.

\section{Metodología}

El enfoque metodológico utilizado para alcanzar los objetivos propuestos de esta investigación está centrado en un estudio cuantitativo descriptivo de corte transversal, que conforme Sampieri et al. (2014 p. 92), este tipo de metodología “busca especificar propiedades y características importantes de cualquier fenómeno que se analice. Describe tendencias de un grupo o población".

Para la realización del estudio, se seleccionó una muestra de 1410 estudiantes, con edades entre 20 y 29 años. Esta cifra corresponde al $52 \%$ de la población $(\mathrm{n}=2711) \mathrm{y}$, de esta, el género femenino representa el $53 \%(\mathrm{n}=748)$ y el masculino, el $47 \%$ (n=662), cantidades que superan la validación de la muestra del $95 \%$ de los estudiantes evaluados $(\mathrm{p}<0,05)$.

A partir de lo anterior, no hay un método estándar diseñado para medir la prevalencia de una exposición o resultado en una población definida y en un punto específico de tiempo. Siendo así, para evaluar el estilo de vida de jóvenes universitarios, existen varios modelos con criterios de validez que utilizan protocolos estandarizados de estilo de vida. Principalmente en el campo de la actividad física, salud y el deporte existen diversas formas, tipos de escalas, 
procedimientos y métodos que describe la importancia y la utilización de herramientas técnicas para aplicar, analizar y evaluar las diversas problemáticas que envuelven los estilos de vida de las personas.

Para esta investigación, se aplicó el formulario estandarizado de autoadministración del pentágono del bien-estar (figura 1), propuesto por Nahas, Barros y Francalacci (2000), que presenta una escala de Likert. Según Fernández (2005), la escala de Likert es un tipo de instrumento de medición o de recolección de datos que se dispone en la investigación social para medir actitudes y conocer el grado de conformidad del encuestado, como también medir situaciones en las que queremos que las personas maticen su opinión.

En este sentido, el cuestionario del pentágono del bien-estar presenta un modelo para evaluar los componentes del estilo de vida que afectan a la salud y el bienestar de las personas. Los componentes evaluados incluyen quince preguntas divididas en cinco criterios de análisis: 1) factores nutricionales; 2) actividad física; 3) conducta preventiva; 4) relaciones sociales; 5) control del estrés.

Nahas et al (2000) consideran que la nutrición, el estrés, la actividad física, el comportamiento preventivo y las relaciones sociales son los componentes que afectan la salud de las personas y están asociados al bienestar psicológico y al desarrollo de las ECNT.

El pentágono del bien-estar es una demostración gráfica de los resultados obtenidos por medio del cuestionario, que es expresada en una figura de un pentágono (estrella), lo cual facilita la visualización de los temas abordados. La escala varía entre 0 y 3 , donde el valor 0 implica la ausencia total del criterio analizado en el estilo de vida; el nivel 1 a veces corresponde al comportamiento; el 2 es casi siempre verdadero en el comportamiento, y el 3 indica una completa realización del comportamiento analizado y que hace parte de su estilo de vida.

Figura 1. Pentágono del bien-estar

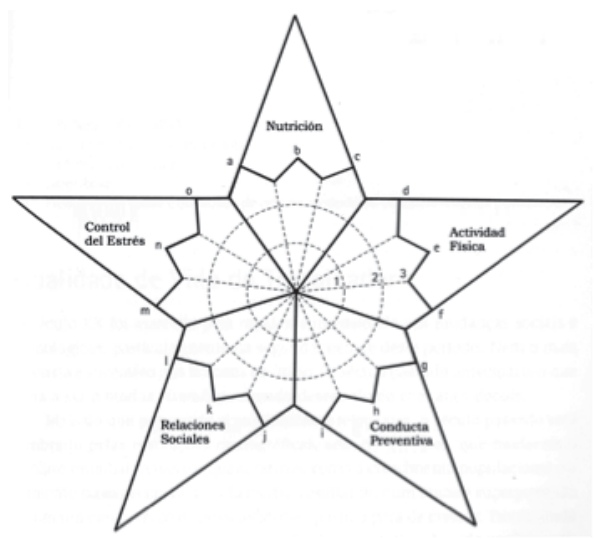

Fuente: Nahas, Barros y Francalacci (2000) 
Una vez terminado el cuestionario, cada ítem puede ser analizado separadamente, o del conjunto, pueden sumarse y obtenerse un valor total del estilo de vida para una validez y calidad estadística. De acuerdo con lo anterior, además de visualizar las respuestas en el pentágono, cada componente evaluado podrá sumar un máximo de 9 puntos y un total de 45 puntos en la sumatoria de las 5 variables evaluadas. En la tabla 1, se puede observar los parámetros de referencia para clasificar el estilo de vida individual

Tabla 1. Clasificación del estilo de vida

\begin{tabular}{cccc}
\hline & \multicolumn{2}{c}{ ÍNDICE } & FÓRMULAS \\
\hline & Positivo & $<2$ & $\frac{=\sum \text { variable individual }}{3}$ \\
ESTILO DE VDA & Regular & $>1.99$ & $=\frac{\sum \text { variable individual total }}{5}$ \\
& & $<1$ & $\frac{5}{2}$ \\
& Negativo & $>0.99$ &
\end{tabular}

Fuente: Nahas, Markus V. (2013). Atividade Física, Saúde e Qualidade de Vida: conceitos e sugestões para um estilo de vida ativo. $6^{\underline{a}}$ edição. Londrina: Midiograf, Brasil

A partir de los resultados, es posible identificar los aspectos positivos y negativos del estilo de vida de las personas, y, asimismo, la información obtenida posibilita la oportunidad de responder o tomar decisiones de intervención que contribuyan para la mejora de la calidad de vida.

Además de la aplicación del cuestionario del pentágono del bien-estar, se colectó información correspondiente a los factores socioeconómicos para correlacionarlos con los estilos de vida de los jóvenes universitarios. En este sentido, mediante una encuesta estructurada, se investigaron los factores y las variables, como el nivel de educación alcanzado por los padres, nivel económico, extracto social, propiedad que habita, información familiar y horas de trabajo semanal.

\section{Resultados}

El análisis de los resultados fue realizado por medio del programa estadístico SPSS, el cual muestra un índice negativo en el factor nutricional (gráfica 1): el $61 \%(n=861)$ no incluye frutas ni verduras en su dieta diaria; el $57 \%(n=804)$ no evita el consumo de alimentos ricos en grasa, y el $25 \%(n=352)$ de la muestra consume menos de cinco alimentos durante el día. 
Gráfica 1. Factor nutricional

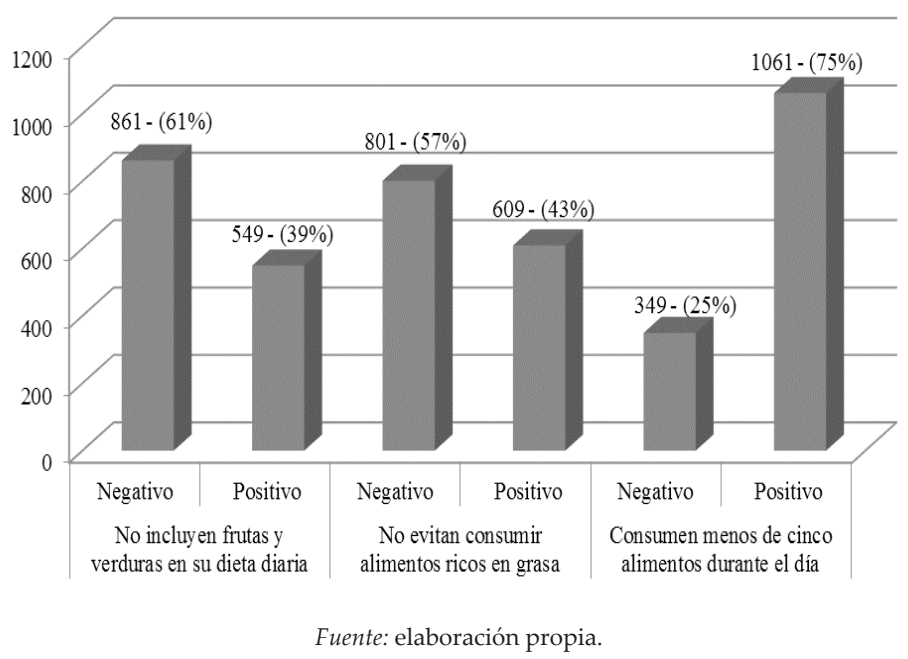

Con relación a la actividad física (gráfica 2), el 47 \% de los estudiantes fueron clasificados como sedentarios y no realizan actividad física de forma moderada o intensa por más de 30 minutos, tres veces a la semana; asimismo, el $35 \%$ no realiza ninguna actividad que involucre ejercicios de fuerza a la semana.

Gráfica 2. Actividad física

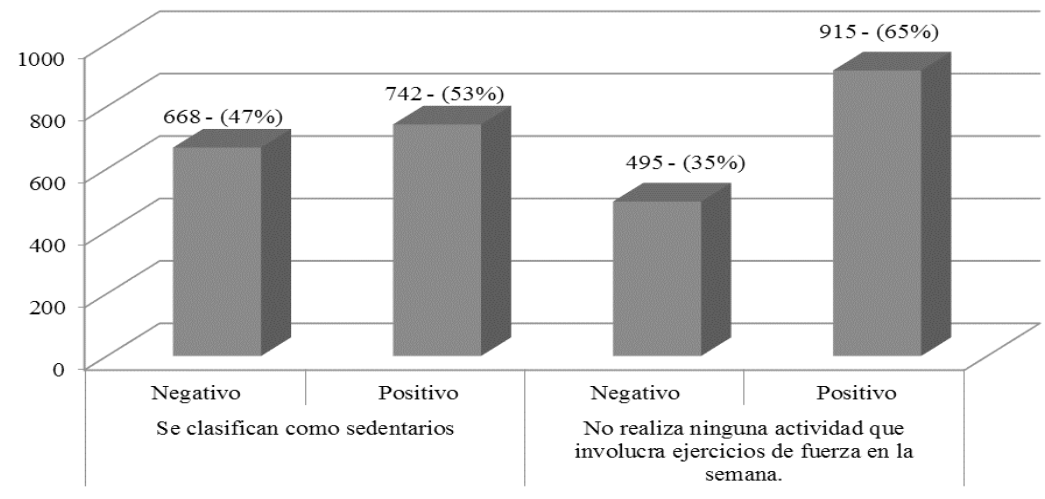

Fuente: elaboración propia.

En la variable de conducta preventiva (gráfica 3), el $73 \%$ de los estudiantes no conoce su estado clínico actual, y el $58 \%$ consume tabaco y alcohol diariamente. 
Gráfica 3. Conducta preventiva

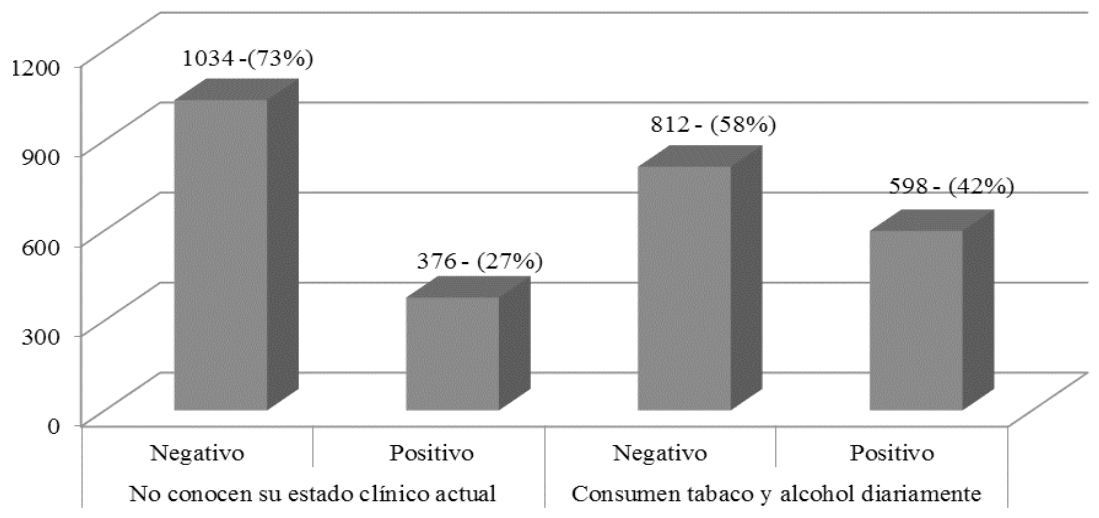

Fuente: elaboración propia.

Por otro lado, el control del estrés (gráfica 4), presento un índice positivo, identificando que el $62 \%$ de los estudiantes dedican un tiempo del mínimo de 5 minutos de su día para realizar pausas activas y relajarse, como también, se observa en los resultados que intentan equilibrar el tiempo libre con el tiempo de trabajo. En cuanto a las relaciones sociales (figura 5) el $92 \%$ están satisfechos con las amistades y participan con frecuencia en reuniones y actividades en la comunidad.

Gráfica 4. Control de estrés y relaciones sociales

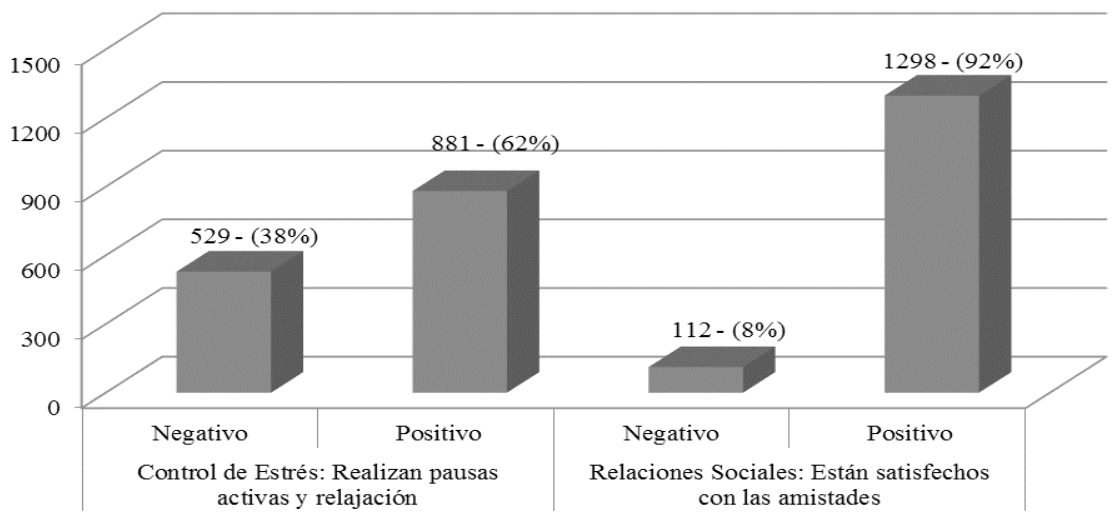

Fuente: elaboración propia.

Teniendo en cuenta lo anterior, se podría decir que estos actos (actividad física y deporte) y actores (estudiantes), conforme propone Bourdieu (1998), mantienen unos esquemas o principios de percepción diferentes, según la forma de sentir;. 
De otro lado, la ausencia de actividad física y el no mantener una conducta preventiva, es lo opuesto a lo que socialmente tiene un alto grado de aceptación, consumir alcohol y tabaco a diario. Por último, en los resultados se observa un alto porcentaje de estudiantes que se sienten cómodos con sus amistades.

A su vez, el estudio socioeconómico (gráfica 5) indica que existe una preferencia por prácticas deportivas individuales, lo que demuestra que el $73 \%$ de los estudiantes tiene como particularidad optar por prácticas en las que no existe un contacto físico. Con relación al estrato social, el $65 \%$ de los estudiantes y familiares están ubicados en un estrato de clase media y el $85 \%$ viven con los padres y familiares. Al mismo tiempo, el $31 \%$ de los estudiantes trabajan y, de estos, el $58 \%$ tiene una carga laboral de más de 40 horas a la semana. Por último, el nivel educativo de los padres revela que el $77 \%$ tienen un nivel educativo alto agrupada en formación universitaria (56 \%) y posgrado (44\%).

Gráfica 5. Estudio socioeconómico

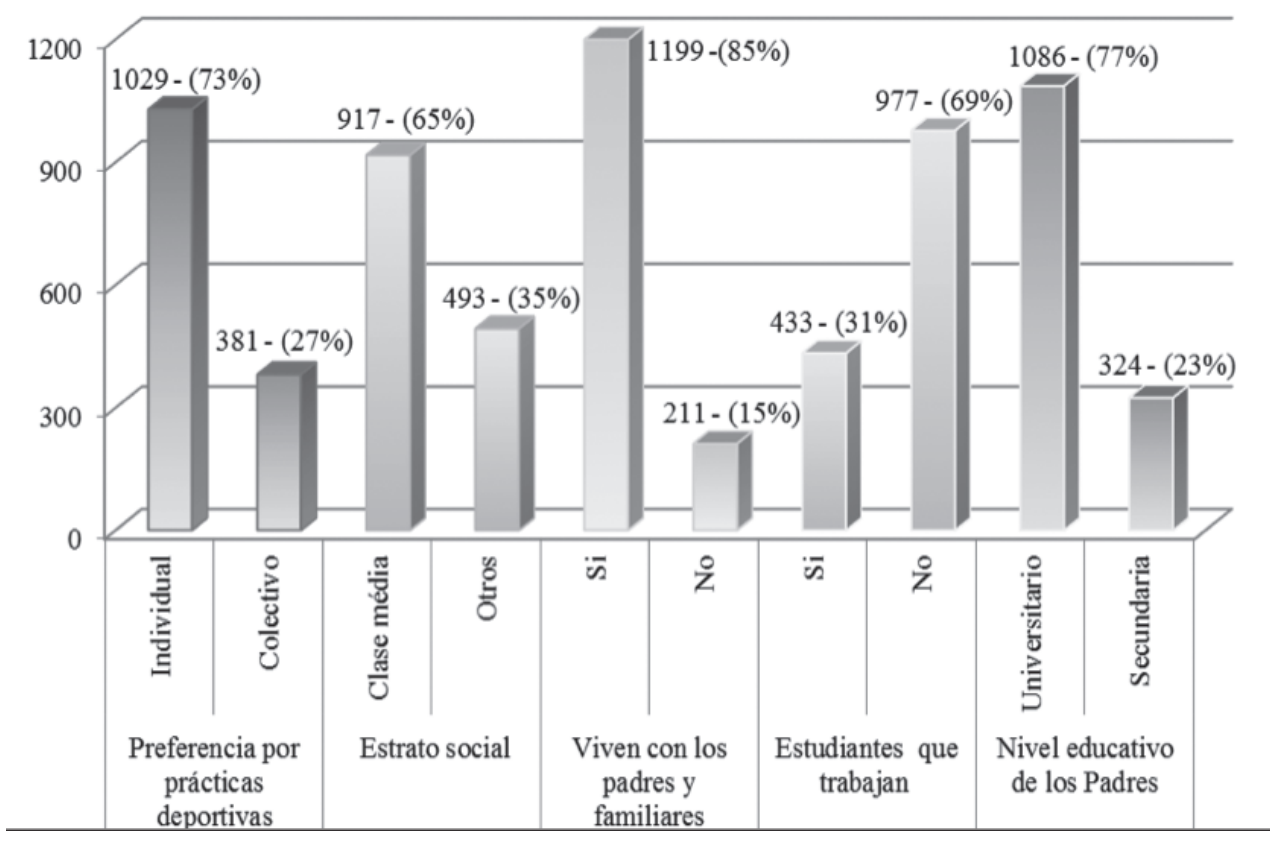

Fuente: elaboración propia.

No existe una relación específica que determine que la actividad física y el deporte depende exclusivamente del factor socioeconómico, también está relacionado con unos sujetos sociales que se diferencian por las distinciones que hacen entre lo distinguido y lo vulgar, el gusto y la clase social (Bourdieu 1998). Lo que para unos es aceptado, para otros es lo inapropiado de los estilos de vida.

Por otra parte, en un mundo globalizado, influenciado por la tecnología de masas, donde el contacto no es físico, sino virtual (a través de chats y redes 
sociales como Whatsapp, Facebook y Twitter), también se refleja un aislamiento corporal en la actividad física y deportiva, que no está relacionado con estudiantes que viven solos.

Por otra parte, un alto porcentaje de los estudiantes conviven con sus padres y provienen de un estrado de clase media, donde su nivel educativo les permite obtener información sobre lo apropiado a su alimentación, que además cuenta con cierta capacidad adquisitiva que les permitiría consumir alimentos denominados nutritivos o sanos.

\section{Conclusiones}

La información recogida en esta investigación permite establecer que en un escenario universitario, donde los estudiantes se encuentran en proceso de formación integral, se percibe que sus estilos de vida en el marco de la actividad física y el deporte no están estrechamente relacionados. No es clara la comprensión entre los factores nutricionales, la actividad física y el guiarse hacia una conducta preventiva; sin embargo, tienen un manejo equilibrado del control del estrés, en tanto que sus relaciones sociales son un factor relevante para ser aceptados por la sociedad.

A partir de este estudio, se evidenció que existe una tendencia de estilos de vida no saludables en los estudiantes de las electivas de actividad física y deporte de la Pontificia Universidad Javeriana. En este sentido, se podría decir que, en esta población, los estilos de vida no saludables podrían ser la causa de las principales enfermedades crónicas no transmisibles, y, a su vez, puede llegar a estimular el detrimento de la salud física en esta población en articular.

Esta investigación arroja resultados preocupantes para una población tan joven, lo que lleva a pensar que en un futuro, si no adoptan medidas de cambio importantes en sus hábitos, un alto porcentaje de estos estudiantes estarán desarrollando uno o más factores de riesgo para la salud.

Los factores socioeconómicos de este estudio demuestran un índice positivo en relación con el estrato social y el nivel educativo de los padres. Esto lleva a reflexionar por qué estos resultados no están reflejados en los estilos de vida de los jóvenes, dado que la gran mayoría de los estudiantes tiene el poder económico considerado bueno conforme el estrato social en el que vive, como también el conocimiento académico y formativo por parte de los padres, lo que podría contribuir con orientaciones a la obtención de un mejor índice en los estilos de vida de los jóvenes.

Sin duda la tendencia actual de promoción de hábitos de vida saludables y de procesos sociales en instituciones de educación superior, están orientados al bienestar y a la calidad de vida. En este sentido, el programa de las electivas 
de actividad física y deporte de la Javeriana brinda a los estudiantes un espacio académico de formación en actividad física, que contribuye al reconocimiento de la cultura física, el bienestar, los hábitos de vida saludables y la promoción de la salud como formación integral del individuo.

Sin embargo, se hace necesario desarrollar nuevas estrategias de intervención en el contexto de las electivas de la Javeriana, para generar un cambio de conducta y adoptar un estilo de vida saludable para la mejora de la calidad de vida.

Teniendo en cuenta la problemática que se está presentando en los jóvenes universitarios, se hace urgente plantear también la intervención del estado a través de políticas públicas que permitan generar una cultura de hábitos de vida saludable, no como un asunto de moda, sino como un espacio institucionalizado desde los niveles básicos de la educación. Tal vez así, al naturalizarse desde edades tempranas, se pueda pensar en una educación integral de las futuras generaciones.

\section{$\mathbf{R}_{\text {eferencias }}$}

American College of Sports Medicine. (2014). ACSM's Guidelines for exercise testing and prescription. Philadelphia, PA: Lipincott Williams \& Wilkins.

Arestedt, L., Persson, C., Benzein, E. (2014). Living as a family in the midst of chronic illness. Scand J Caring Sci, 28(1), 29-37.

Bandura, A. (julio de 1977). Social learning theory. New York: General Learning Press.

Batista, J., Fortiana, J. y Currie, C. (2004). Socio-economic indexes in surveys for comparisons between countries. Social Indicators Research, 67(3). 315-332.

Bourdieu, P. (1998). El habitus y el espacio de los estilos de vida. En La distinción. Barcelona: Taurus.

Daviglus, M., Stamler, J., Pirzada, A., Yan, L., Garside, D., Liu, K., ...Greenland, P. (2004). Favorable cardiovascular risk profile in young women and longterm risk of cardiovascular and all-cause mortality. Jama, 292(13):1588-1592. DOI:10.1001/jama.292.13.1588.

Díaz, M., Tovar, M. y Torres, A. (Enero-marzo, 2015). Estilos de vida y autoimagen en estudiantes de colegios oficiales en Chía, Cundinamarca. Revista Pediatría,. 48(1), 15-20.

Fernández de Pinedo, I. (2005). Construcción de una escala de actitudes tipo Likert. Madrid: Instituto Nacional de Seguridad e Higiene en el Trabajo. 
Garzón, N. y Rodríguez, D. (2012). Desarrollo de intervenciones en actividad física en Brasil y Colombia. Bogotá: Universidad Nacional de Colombia.

Gluckman, P. D., Hanson, M. A., Cooper, C., Thornburg, K. L. (Julio de 2008). Effect of in utero and early-life conditions on adult health and disease. $N$ Engl J Med. 359(1), 61-73. DOI: 10.1056/NEJMra0708473.

Wang, G., Walker, S. O., Hong, X., Bartell, T. R. y Wang, X. (Febrero de 2013). Epigenetics and early life origins of chronic noncommunicable diseases. Journal of Adolescent Health, 52(2), 14-21. DOI: 10.1016/j. jadohealth.2012.04.019.

Hernando, A., Oliva, A. y Pertegal, M. (Abril de 2013). Diferencias de género en los estilos de vida de los adolescentes. Psychosocial Intervention, 22(1), 15-23. DOI: 10.5093/in2013a3.

Krupp, M. y Chatton, M. (2014). Current medical diagnosis E treatment. Los Altos: Lange Medical.

Martín, M. y Velarde, O. (2001). Informe de juventud 2000. Madrid: Ministerio de Asuntos Sociales.

Moscoso, D., Martín, M., Pedrajas, N. y Sánchez, R. (2013). Sedentarismo activo. Ocio, actividad física y estilos de vida de la juventud española. Arch Med Deporte, 30(6): 341-437.

Nahas, M. V., Barros, M. V. G. y Francalacci, V. (2000). O pentáculo do bem-estar: base conceitual para avaliação do estilo de vida de indivíduos ou grupos. Revista Brasileira de Atividade Física \& Saúde, 5(2), 48-59.

Nahas, M. (2013). Atividade física, saúde e qualidade de vida: conceitos e sugestões para um estilo de vida ativo (6. ${ }^{\mathrm{a}}$ ed.). Londrina: Midiograf.

Organización Mundial de la Salud. (2005). Preventing chronic diseases: A vital investment. Ginebra: Organización Mundial de la Salud.

Organización Mundial de la Salud. (2008). Una guía de enfoques basados en población para incrementar los niveles de actividad física: aplicación de la estrategia mundial sobre régimen alimentario, actividad física y salud. Ginebra: Organización Mundial de la Salud.

Organización Mundial de la Salud. (2013). Directrices: Ingesta de sodio en adultos y niños. Ginebra: Organización Mundial de la Salud.

Organización Mundial de la Salud. (2013). Action plan for the global strategy for the prevention and control of noncommunicable diseases. Ginebra: Organización Mundial de la Salud. 
Vidarte, J., Vélez, C. y Parra, J. (Junio de 2012). Niveles de sedentarismo en población de 18 a 60 años. Manizales, Colombia. Rev. Salud Pública, 14(3), 417-428.

Zea, A., León, H., Botero, D., Afanador, H. y Pinzón, L. (Agosto de 2014). Factores de riesgo cardiovascular y su relación con la composición corporal en estudiantes universitarios. Rev. Salud Pública, 16(4), 505-515. 